\title{
The risk factors for suboptimal CD4 recovery in HIV infected population: an observational and retrospective study in Shanghai, China
}

\author{
Fengdi Zhang ${ }^{1}$, Meiyan Sun ${ }^{1}$, Jianjun Sun ${ }^{1}$, Liqian Guan ${ }^{1}$, Jiangrong Wang ${ }^{1}$, Hongzhou Lu ${ }^{1,2,3, *}$ \\ 'Department of Infectious Disease, Shanghai Public Health Clinical Center, Fudan University, Shanghai, China; \\ ${ }^{2}$ Department of Infectious Disease, Huashan Hospital Affiliated to Fudan University, Shanghai, China; \\ ${ }^{3}$ Department of Internal Medicine, Shanghai Medical College, Fudan University, Shanghai, China.
}

\begin{abstract}
Summary Although the initiation of antiretroviral therapy (ART) has promoted the reconstitution of CD4+ T-cell count in the HIV infected population, not all patients can achieve the normalization of their immunologic functions. We analysed the variables associated with immunologic recovery, which is commonly regarded as the increase of CD4 to 350 cell $/ \mu \mathrm{L}$ after a year of ART. We collected data from 3,485 patients attending a university-based HIV clinic from June 2005 to July 2014 in Shanghai, China. Logistic regression test was performed to analyse the risk factors for suboptimal CD4+ recovery following yearlong ART. The CD4+ T-cell of 723 participants $(41.5 \%$ of the 1744 subjects) showed more than $350 \mathrm{cell} / \mu \mathrm{L}$ after one year of ART. Compared with baseline CD4 $>350 \mathrm{cell} / \mu \mathrm{L}$, patients with baseline CD4 $\leq 200$ cell $/ \mu \mathrm{L}$ or $200<\mathrm{CD} 4 \leq 350 \mathrm{cell} / \mu \mathrm{L}$ were $42.6,4.5$ times more likely to be incomplete CD4 recovery, respectively. The risk of suboptimal immunologic recovery among patients with regimen including AZT or d4T were 2.1, 2.4 times higher compared with TDF, respectively. In our study, between optimal CD4 recovery group and suboptimal recovery group, there were no significant differences in age, gender, marital status, transmission routes, WHO stage, and CD4 recovery rates. As for the dynamic CD4 change, we found the CD4 recovery rates were $49.9 \%$ and $61.8 \%$ in the second and third year of ART, respectively. Patients who had a low level of CD4+ T-cell count $(<200$ cell/ $\mu \mathrm{L}$ ) during the initiation of ART exhibited more difficulties recovering to a normal level. Furthermore, the regimen, including AZT or d4T, was not beneficial to CD4 recovery. So, more efforts should be made to guarantee the early diagnosis and timely treatment for HIV/ AIDS patients, and simultaneously optimize antiretroviral therapy.
\end{abstract}

Keywords: HIV, antiretroviral therapy, CD4 recovery, suboptimal immunologic response, risk factors

\section{Introduction}

For many years, HIV infection has been a serious public health burden worldwide (1-6). The CD4+ T cell plays a critical role in the pathogenesis of the HIV infection. As the main target cells of HIV, after being infected,

*Address correspondence to:

Dr. Hongzhou Lu, Shanghai Public Health Clinical Center, Fudan University, No.2901, Caolang Road, Jinshan District, Shanghai, China.

E-mail: luhongzhou@fudan.edu.cn
CD4+ T-cell count reduces significantly and leads to the deconstruction of T-cell immune response, which consequently causes immunodeficiency and increases incidences of opportunistic infections and tumours. Two hundreds cells per microliter of CD4 count is a critical point in clinical practice because patients with CD4 counts above 200 cell $/ \mu \mathrm{L}$ have lower risk of clinical events $(7,8)$.

Highly active antiretroviral therapy (HAART) for HIV infection is among the greatest successes of modern medicine, having remarkably prolonged the expected life span of HIV-infected individuals (9-13). With HAART, HIV infection can be prevented from 
transforming into clinical AIDS. As a result, what was commonly considered as a fatal disease is now regarded as a chronic condition. However, after the initiation of HAART, the outcome of some patients remained unsatisfactory, with the possible occurrence of virologic failure and suboptimal immunologic response (SIR). Currently, there is no consensus on the definition of suboptimal immunologic response, but certain studies have recommended that immunologic response failure can be identified as not managing to increase CD4 counts to more than 350 cell $/ \mu \mathrm{L}$ or 500 cell $/ \mu \mathrm{L}$ within a specific time period (e.g., 4 to 7 years) (7).

Given the fact that incomplete CD4 recovery still occurs after the initiation of HAART, risk factors for this outcome should be analysed. According to published data $(8,14-18)$, predictors for suboptimal CD4 recovery are as follows: $\mathrm{CD} 4<200 \mathrm{cell} / \mu \mathrm{L}$ at the initiation of ART, older age, co-infected with hepatitis $\mathrm{C}$ virus, HIV-2, human T-cell leukaemia virus, regimen of ART, persistent immune activation, loss of the regenerative potential of the immune system and concomitant medical conditions. However, limited studies mainly focused on the risk factors for incomplete immunologic recovery in China, which is a resource-limited place. Therefore, in this context, we conducted an observational and retrospective study to evaluate the influence factors of CD4+ T-cell count recovery rate of the HIV positive population in East China.

\section{Materials and Methods}

\subsection{Study design, subjects and inclusion criteria}

We conducted the cohort study of HIV-infected patients who attended the clinic affiliated with Fudan University, from June 2005 to July 2014 in Shanghai, China. Participants were only enrolled if they were HIV positive and on stable ART. Their CD4 count and virus load were not considered. Besides, all participants were not infected with the hepatitis B virus or the hepatitis $\mathrm{C}$ virus. Our study conformed to the principles of the Declaration of Helsinki and the current Guideline of Diagnosis and Treatment of AIDS. Every enrolled patient was offered a written informed consent form.

\subsection{Data collection}

The patients' data was firstly collected from their charts, and afterwards a professional staff, majoring in Hospital Information Management, manually entered the data into a national electronic database. In addition, the clinicians routinely checked the data to ensure accuracy and consistency.

\subsection{ART therapy}

ART management at our hospital complied with the
Chinese National Antiretroviral Therapy Guidelines. Based on the present situation in China, first-line regimen includes a combination of two nucleoside reverse transcriptase inhibitors (NRTIs): lamivudine (3TC) and zidovudine (AZT) or tenofovir (TDF) or stavudine $(\mathrm{d} 4 \mathrm{~T})$ and a nonnucleotide reverse transcriptase inhibitor (NNRTI): efavirenz (EFV) or nevirapine (NVP). In the second-line regimen, a protease inhibitor, preferably lopinavir/ritonavir $(\mathrm{LPV} / \mathrm{r})$, is used to substitute for the nonnucleotide reverse transcriptase inhibitor. Approximately every three months, we monitored the ART therapy with CD4+ T-cell count. Apart from the T lymphocyte immunity test, the blood routine examination and blood biomedical assay were also conducted. The patients received the virus load test at the sixth month of ART, and then the test was to be conducted annually. To observe the changing trend of CD4 counts, we gathered follow-up data every three months during the first year, and every six months from the second to the third year.

\subsection{CD4+T-cell count measurement}

Blood samples for CD4+ T-Cell count measurement were analysed with CYTOMICS-FC500 at the Shanghai Public Health Clinical Centre affiliated with Fudan University. In our study, we defined the baseline CD4+ T cell count as the most recent test performed within one month before ART was initiated.

\subsection{Analysis and statistics}

Data analysis was conducted by the IBM SPSS version 19.0 (IBM SPSS, Inc., Armonk, NY, USA). Continuous variables were described using mean and standard deviation (SD), while categorical variables were described by numbers and percentages. The chi square test was used for categorical variables and the $\mathrm{t}$ test was used for continuous variables. We used the logistic regression test to analyse the risk factors for suboptimal CD4 recovery after HAART initiation. The confounding factors included: age, gender, marital status, infection route, treatment regimen, WHO stages, initiation of ART timing and baseline CD4 cell counts. All hypothesis testing was two-sided, with a level of $\alpha$ $=0.05$.

\section{Results}

\subsection{Characteristics of the participants}

1,744 participants, with a mean age of 37.3 years old (min 18, max 88), were enrolled in this study (Figure 1). Most subjects were male and nearly half of the participants were single, while $40 \%$ of the participants were married or cohabiting with others. When it comes to the infection routes, the majority of participants 


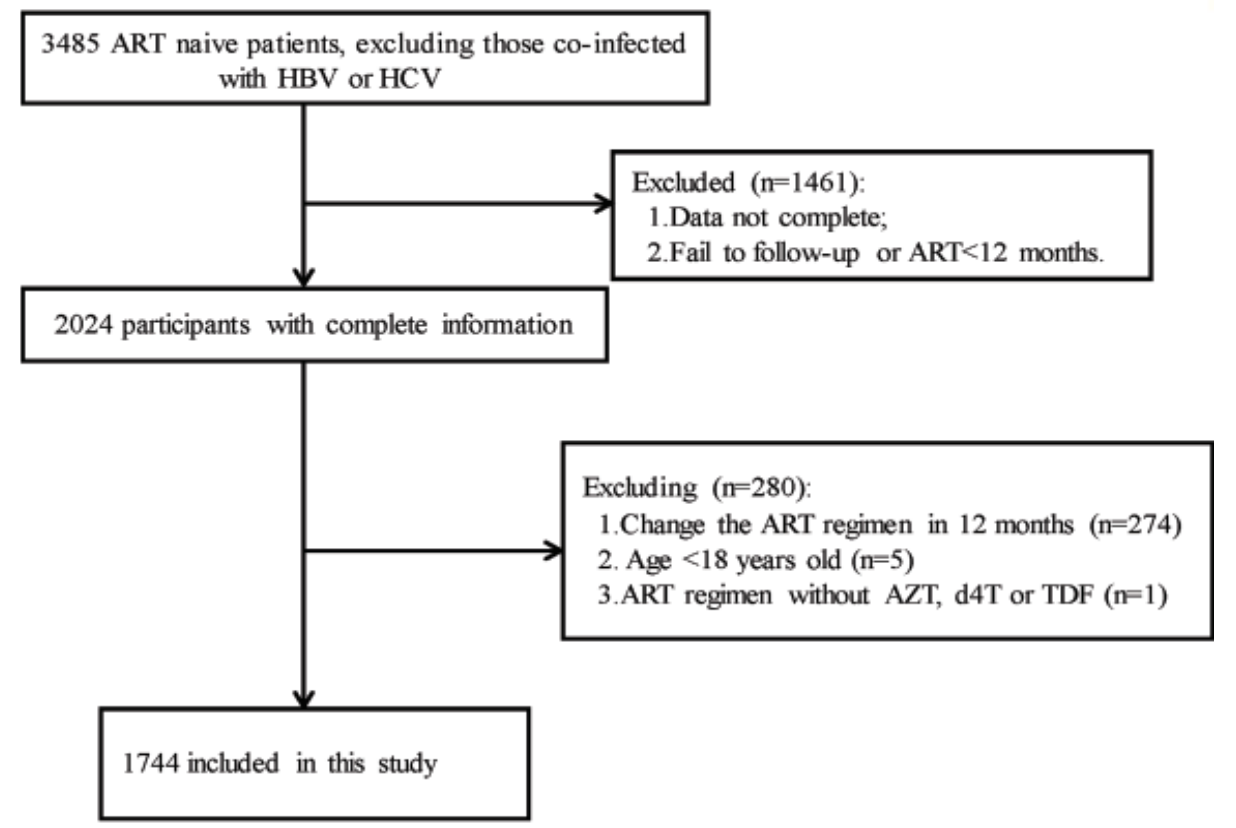

Figure 1. Flow chart of the study. Inclusion criteria of the participants in HIV voluntary consult and test clinic of SHAPHC.

were infected via sexual transmission: $1,017(58.3 \%)$ were homosexual men (MSM) and 395 (22.6\%) were heterosexual transmission. Blood transfusion and intravenous drug users (IDU) accounted for $1.7 \%$ and $1.5 \%$, respectively. Those who did not know how they became infected with HIV accounted for $15.8 \%$. Of all the participants, a fairly large proportion was at the third WHO stage. As for the timing of ART initiation, 1,187 subjects (68.1\%) started ART within six months from the time they were diagnosed as being HIV positive. The mean CD4 count of our participants before they received ART was about 202 cell $/ \mu \mathrm{L}$. After one year of ART, the mean CD4 count was 340 cell/ $\mu \mathrm{L}$, with an increase of some 140 cell $/ \mu \mathrm{L}$. The most commonly used regimen in our study was two NRTIs + 1 NNRTI, in which AZT + 3TC + EFV was received by more than half of the participants (Table 1).

\subsection{Prevalence and risk factors for suboptimal CD4+ T-cell recovery}

One year after the initiation of ART, the CD4+ T-cell count of 723 subjects (41.5\% of all 1,744 participants) was above 350 cell $/ \mu \mathrm{L}$, which was defined as the recovered group. While the CD4+ T-cell count of the remaining 1,021 participants failed to achieve the same level; it was defined as the unrecovered group. There was no significance in age between the two above groups $(p=0.207)$. There was also no difference in gender $(p=0.461)$ and marital status $(p=0.061)$ between the two above groups. However, through the chi-square test, we found that the transmission routes of the two groups had discrepancy $(p<0.001)$. Specifically, the rate of those infected with HIV
Table 1. Characteristics and analysis of the demographics of the 1,744 participants

\begin{tabular}{lc}
\hline Variable & Number (\%) in 1,744 participants \\
\hline Age at start of ART (Mean \pm S.D.) & $37.3 \pm 12.0$ \\
Gender & \\
Male & $1588(91.1)$ \\
Female & $156(8.9)$ \\
Marital status & \\
Single & $901(51.7)$ \\
Married/cohabiting & $692(39.7)$ \\
Divorced/separated & $120(6.9)$ \\
Widowed & $15(0.9)$ \\
Unknown & $16(0.9)$ \\
Infectious routes & \\
Blood transfusion & $30(1.7)$ \\
IDU & $26(1.5)$ \\
MSM & $1017(58.3)$ \\
Heterosexual transmission & $395(22.6)$ \\
Unknown & $276(15.8)$ \\
CD4 count (Mean \pm S.D.) & \\
At baseline & $202.4 \pm 166.1$ \\
At 1 year of ART & $339.7 \pm 181.5$ \\
Timing of the initiation of ART & \\
Less than 6 months & $1187(68.1)$ \\
7-12 months & $151(8.7)$ \\
13-24 months & $173(9.9)$ \\
More than 24 months & $233(13.4)$ \\
ART regimen & $1039(59.6)$ \\
AZT+3TC+EFV & $66(3.8)$ \\
d4T+3TC+NVP & $195(11.2)$ \\
d4T+3TC+EFV & $247(14.2)$ \\
TDF+3TC+EFV & $22(1.3)$ \\
TDF+3TC+LPV/r & $118(6.8)$ \\
AZT+3TC+NVP & $42(2.4)$ \\
AZT+3TC+LPV/r & $15(0.9)$ \\
Others & $12(0.7)$ \\
WHO stage & $26(1.5)$ \\
Stage 1 & $1282(73.5)$ \\
Stage 2 & $424(24.3)$ \\
Stage 3 & \\
Stage 4 & \\
\hline
\end{tabular}


Table 2. The risk factors for incomplete CD4 recovery after the first year's ART by logistic regression test

\begin{tabular}{|c|c|c|c|c|c|c|c|}
\hline \multirow{2}{*}{ Factors } & \multirow{2}{*}{$\begin{array}{c}\text { Recovered } \\
(n=723)\end{array}$} & \multirow{2}{*}{$\begin{array}{l}\text { Unrecovered } \\
(n=1021)\end{array}$} & \multirow{2}{*}{$p$ value } & \multicolumn{2}{|c|}{ Univariate Analysis } & \multicolumn{2}{|l|}{ Multivariate Analysis } \\
\hline & & & & Odds ratio $(95 \% \mathrm{CI})$ & $p$ value & Adjusted OR (95\% CI) & $p$ value \\
\hline Age group (Mean \pm S.D.) & $36.0 \pm 12.0$ & $38.3 \pm 12.0$ & $0.207^{\#}$ & & & & \\
\hline $18-44$ yr., $n(\%)$ & $569(78.7)$ & $718(70.3)$ & & $0.708(0.464-1.080)$ & 0.109 & & \\
\hline $45-59$ yr., $n(\%)$ & $118(16.3)$ & $239(23.4)$ & & $1.111(0.699-1.766)$ & 0.657 & & \\
\hline$\geq 60$ yr., $n(\%)$ & $36(5.0)$ & $64(6.3)$ & & Reference & & & \\
\hline Gender & & & 0.461 & & & & \\
\hline Male, $n(\%)$ & $654(90.5)$ & $934(91.5)$ & & $1.124(0.807-1.565)$ & 0.490 & & \\
\hline Female, $n(\%)$ & $69(9.5)$ & $87(8.5)$ & & Reference & & & \\
\hline Married status & & & 0.061 & & & & \\
\hline Married/cohabiting, $n(\%)$ & $268(37.1)$ & $424(41.5)$ & & 1. $184(0.974-1.439)$ & 0.090 & & \\
\hline Unmarried/no cohabiting, $n(\%)$ & $455(62.9)$ & $597(58.5)$ & & & & & \\
\hline Infectious route & & & $<0.001$ & & & & \\
\hline MSM, $n(\%)$ & $455(62.9)$ & $562(55.0)$ & & Reference & & Reference & \\
\hline Heterosexual transmission, $n(\%)$ & $156(16.0)$ & $239(23.4)$ & & $1.227(0.969-1.555)$ & 0.089 & $0.905(0.665-1.231)$ & 0.525 \\
\hline Unsexual transmission, $n(\%)$ & $112(15.5)$ & $220(21.5)$ & & $1.548(1.196-2.004)$ & 0.001 & $0.827(0.578-1.185)$ & 0.302 \\
\hline Treatment regimen & & & $<0.001$ & & & & \\
\hline Including AZT, $n(\%)$ & $526(72.8)$ & $674(66.0)$ & & $1.409(1.084-1.831)$ & 0.010 & $2.052(1.480-2.846)$ & $<0.001$ \\
\hline Including d4T, $n(\%)$ & $53(7.3)$ & $215(21.1)$ & & $4.490(3.064-6.581)$ & $<0.001$ & $2.400(1.531-3.762)$ & $<0.001$ \\
\hline Including TDF, $n(\%)$ & $144(20.0)$ & $132(12.9)$ & & Reference & & Reference & \\
\hline WHO stage & & & 0.157 & & & & \\
\hline Stage $1,2, n(\%)$ & $20(2.8)$ & $18(1.8)$ & & Reference & & & \\
\hline Stage $3,4, n(\%)$ & $703(97.2)$ & $1003(98.2)$ & & $1.574(0.827-2.997)$ & 0.168 & & \\
\hline Timing of the initiation of ART & & & 0.005 & & & & \\
\hline Less than 6 months, $n(\%)$ & $465(64.3)$ & $722(70.7)$ & & $1.354(1.105-1.660)$ & 0.003 & $1.116(0.879-1.418)$ & 0.368 \\
\hline More than 6 months, $n(\%)$ & $258(35.7)$ & $299(29.3)$ & & Reference & & Reference & \\
\hline Baseline CD4 count & & & $0.033^{\#}$ & & & & \\
\hline$($ cell $/ \mu \mathrm{l})$ mean \pm S.D. & $285.4 \pm 196.1$ & $143.7 \pm 107.5$ & & & & & \\
\hline $\mathrm{CD} 4 \leq 200, n(\%)$ & $115(15.9)$ & $682(66.8)$ & & $47.552(24.675-91.641)$ & $<0.001$ & $42.597(21.927-82.752)$ & $<0.001$ \\
\hline $200<\mathrm{CD} 4 \leq 350, n(\%)$ & $518(71.6)$ & $328(32.1)$ & & $5.155(2.715-9.788)$ & $<0.001$ & $4.519(2.367-8.627)$ & $<0.001$ \\
\hline $\mathrm{CD} 4>350, n(\%)$ & $90(12.4)$ & $11(1.1)$ & & Reference & & Reference & \\
\hline
\end{tabular}

\# These $\mathrm{p}$ values were calculated by $t$-test. The others were analyzed by chi-square test.

through MSM was slightly higher in the recovered group than in the unrecovered group $(62.9 \% \mathrm{vs}$. $55.0 \%)$. On the contrary, the rate of heterosexual transmission and unsexual transmission, $16.0 \%$ and $15.5 \%$, respectively, in the recovered group was lower than that of the uncovered group, $23.4 \%$ and $21.5 \%$, respectively.

The rate of different ART regimens was also diverse between the recovered group and the unrecovered group: the regimen included AZT (72.8\% vs. 66.0\%), d4T (7.3\% vs. $21.1 \%)$ and TDF (20.0\% vs. 12.9\%). Surprisingly, no difference was found in the WHO stage between the two groups $(p=0.157)$. In view of the timing of ART initiation, the proportion of participants who initiated ART earlier ( $\leq 6$ months from being diagnosed with HIV infection) was approximately $64 \%$ in the recovered group as compared to about $71 \%$ in the unrecovered group. The baseline CD4 count of the two groups showed significant differences $(p=0.033)$.

When we conducted the univariate logistic analysis, we found that among all the variables, only the ART regimen and the baseline $\mathrm{CD} 4+\mathrm{T}$-cell count were associated with suboptimal CD4+ T-cell recovery. Furthermore, the multivariate analysis showed that the lower baseline CD4+ T-cell count and the regimen including AZT and d4T, were higher risk factors for CD4+ T-cell unrecovery (Table 2).

\subsection{Follow-up situation of participants}

Among all the 1,744 participants, the CD4+ T-cell count of only 101 participants was above 350 cell $/ \mu \mathrm{L}$ at baseline. After a year of ART, the CD4 count of 723 subjects was above this level. The data corresponding to the second year of ART decreased by 793 because some patients had not reached the second year of their ART, or we failed to follow up due to certain personal or impersonal factors. In the remaining 951 subjects, 476 people had a CD4+ T-cell count above 350 cell/ $\mu \mathrm{L}$. We had 455 subjects with complete information at the third year of ART, and among them 281 participants had CD4 count above 350 cell $/ \mu \mathrm{L}$ (Figure 2A).

At the baseline, only $5.8 \%$ of the participants had a CD4+ T-cell count above 350 cell $/ \mu \mathrm{L}$ and the CD4 recovery rate increased to $41.5 \%$ after a year of ART. After eliminating the participants with missing data, the CD4 recovery rate increased to $49.9 \%$ and $61.8 \%$ in the second and third years of ART, respectively (Figure 2B).

\section{Discussion}

In this study, we analysed the risk factors for incomplete CD4 recovery in the HIV-infected population of East China. We found that the ART regimen and the baseline 

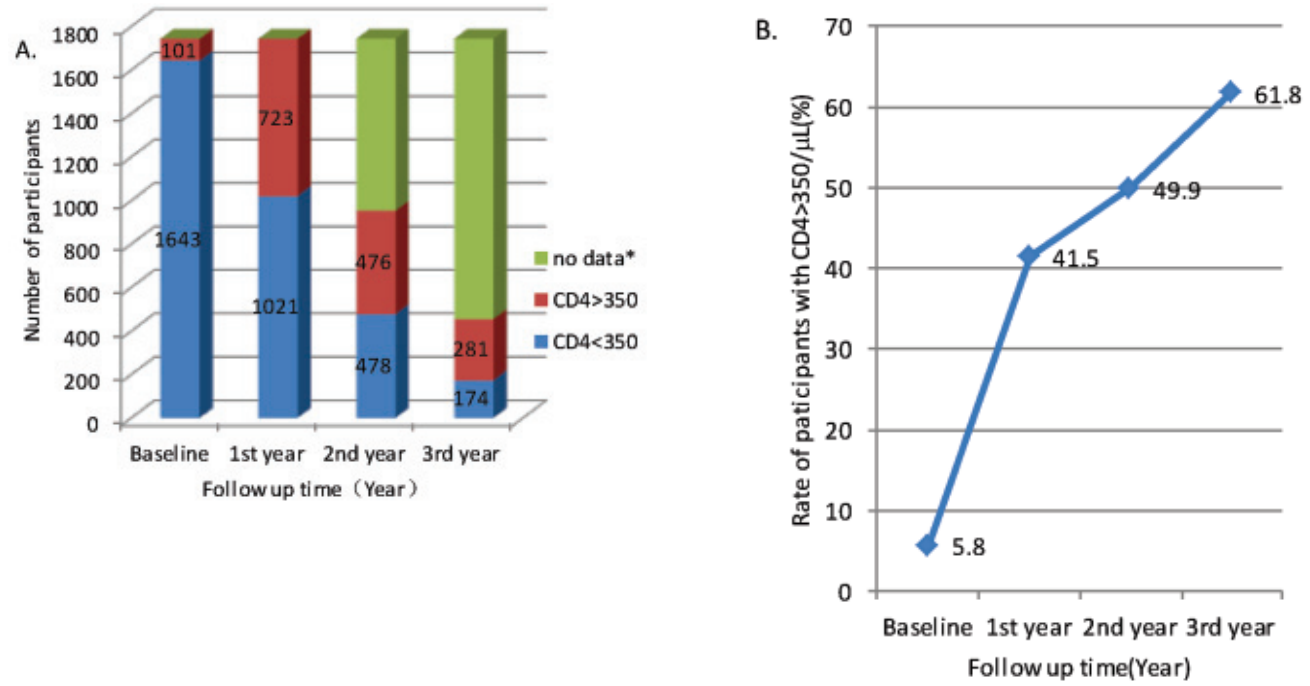

Figure 2. Review of participants' follow-up for 3 years. (A), Proportion of participants with different CD4 level or without data. (B), Trend of CD4 recovery rate. * Participants who had received ART less than 3 years or without follow-up information.

of CD4+ T-cell count could impact immunological restoration. AZT, d4T and TDF are commonly used among HIV infectors in China. However, compared with TDF, the regimen including AZT and d4T were more likely to suppress the recovery of CD4+ T-cell. Those with higher baseline CD4+ T-cell count are more likely to achieve full immunological recovery.

Through statistical analysis, we drew the same conclusion that the lower baseline CD4 count is adverse to immunologic restoration. Compared with those whose baseline CD4 > 350 cell $/ \mu \mathrm{L}$, patients with CD4 between 200 and 350 cell $/ \mu \mathrm{L}$ or CD4 $<200$ cell $/ \mu \mathrm{L}$ at ART initiation were more likely to have suboptimal immunologic responses, with the odds ratio of 42.597 (95\% CI: $21.927-82.752)$ and 4.519 (95\% CI: $2.367-$ $8.627)$, respectively. All of the above results were similar with the results about predictors for incomplete CD4 recovery after HAART initiation mentioned in the US AIDS guidelines (7).

Our study also found that ART regimen could impede CD4 recovery to some extent. As different ART regimens are applied in different countries, we studied ART regimens concerning 3TC, d4T, AZT, and TDF, which are commonly used for patients and free of charge in China. Compared with the ART regimen including TDF, we found that the regimens including AZT and $\mathrm{d} 4 \mathrm{~T}$ impeded CD4 recovery, and the odds ratio were 2.052 (95\% CI: $1.480-2.846)$ and $2.400(95 \%$ CI: 1.531-3.762), respectively. Our result is similar to the study conducted in 2006 by Gallant JE, et al. and the study conducted in Senegal by G. Batista, et al $(8,19)$. The incomplete CD4 recovery among patients with regimen including AZT or d4T can be explained by the fact that both these drugs have myelotoxicity, and there is a smaller increase of total lymphocyte count when AZT and d4T are included in an ART regimen (20-22). Li TS' study has demonstrated that the thymus function of adults will gradually decline with the increase of age. It is well known that the maturity of $\mathrm{T}$ lymphocytes is in the thymus, thus aging undoubtedly affects CD4+ T-cell count (23). However, our univariate analysis showed that age was not associated with CD4+ T-cell recovery, which was inconsistent with the results in the guideline of the USA. The main reason is that the number of elder subjects ( $>60$ years) was not large enough comparing with other age groups (chi-square test, $p=0.246$ ).

Nowadays, there is no specific standard for the early therapy of HIV/AIDS, but the current study indicated that early therapy is superior to delayed therapy, resulting in better virus suppression, less confection with other diseases, lower morbidities and better CD4+ T-cell recovery (24). It is difficult to determine when these patients became infected with HIV, so we substituted the diagnosis date for the infection time. We defined early therapy as initiating ART regimen less than six months from the date they received diagnosis reports, and delayed therapy was defined as more than six months from the time of confirmed diagnosis. However, in our study, we found that the timing of ART initiation was not a risk factor for incomplete $\mathrm{CD} 4+\mathrm{T}$-cell recovery (univariate analysis, $\mathrm{OR}=1.116$, $95 \%$ CI: $0.879-1.418, p=0.368)$. Therefore, a credible way needs to be established to verify the exact time of infection for the accurate analysis of the relationship between the timing of ART initiation and treatment efficacy.

Although a high degree of concern is paid to the CD4+ T-cell recovery in the field of HIV/AIDS research, various conclusions have been obtained from different studies. An urban cohort study conducted in sub-Saharan Africa indicated that if the CD4 recovery criterion is stipulated at the cut-off of $200 \mathrm{cell} / \mu \mathrm{L}$, then the recovery rates would be $57 \%, 58 \%$, and $81 \%$ 
at the sixth, twelfth, and twenty-forth months of ART, respectively (25). Another study conducted in Senegal declared that if suboptimal immune reconstitution is defined as the increase of CD4 count less than 50 cell/ $\mu \mathrm{L}$ in patients with virologic suppression more than six months, then the recovery rate would be $84.5 \%$ after a year of ART (8). Our cohort study showed that the CD4 recovery rates were $41.5 \%, 49.9 \%$, and $61.8 \%$ after twelve, twenty-four, and thirty-six months of ART, separately. Apparently, in the same follow-up time, the immune reconstitution rate will be higher if the recovery standard is set at a lower point. Obviously, with the longer period of having ART, the immune reconstitution of patients is more promising.

However, our study still had two limitations. Firstly, not all of the participants had their HIV-RNA levels measured at the baseline due to the high cost of the test. This test was free of charge after a year of ART. 2,241 patients out of the 3,485 participants had HIV-RNA level tests, with 2,179 people having their HIV-RNA levels lower than $1.0 \times 10^{3}$ copies $/ \mathrm{mL}$. Therefore, we can come to the conclusion that we had a satisfactory virologic suppression (97.2\%). Secondly, the information of some patients was lost or incomplete due to certain reasons during the second and third years from the initiation of ART. In turn, this set limitations for us to observe the dynamic changes in CD4 recovery of all of the participants and evaluate the exact recovery rate.

In conclusion, we carried out an observational, retrospective and cohort research to study the risk factors for suboptimal CD4 recovery in the HIV infected population of Shanghai, China. We discovered that patients who had a low level of CD4+ T-cell count $(<200$ cell $/ \mu \mathrm{L})$ at the initiation of ART had more difficulties recovering to the normal level. Furthermore, the regimen including AZT or d4T impedes CD4 recovery. Actually, the CD4 recovery rate is not satisfactory in our settings. To decrease the incidence of opportunistic infection and mortality in the HIV infected population, we should endeavour to facilitate the normalization of their CD4+ T-cell count. At the same time, more efforts should be made to guarantee the early diagnosis and early treatment for HIV/AIDS patients as well as optimizing antiretroviral therapy.

\section{Acknowledgements}

This study was supported by key research grants from the Ministry of Science and Technology and the People's Republic of China (2012ZX09303013 and 2014AA021403).

\section{References}

1. Oram S, Stockl H, Busza J, Howard LM, Zimmerman C. Prevalence and risk of violence and the physical, mental, and sexual health problems associated with human trafficking: Systematic review. PLoS Med. 2012; 9:e1001224.

2. Turan JM, Nyblade L. HIV-related stigma as a barrier to achievement of global PMTCT and maternal health goals: A review of the evidence. AIDS Behav. 2013; 17:2528-2539.

3. Bobkova M. Current status of HIV-1 diversity and drug resistance monitoring in the former USSR. AIDS Rev. 2013; 15:204-212.

4. Hoare J, Ransford GL, Phillips N, Amos T, Donald K, Stein DJ. Systematic review of neuroimaging studies in vertically transmitted HIV positive children and adolescents. Metab Brain Dis. 2014; 29:221-229.

5. Gelu-Simeon M, Sobesky R, Haim-Boukobza S, Ostos M, Teicher E, Fontaine H, Salmon-Ceron D, Meyer L, Trinchet JC, Paule B, Samuel D, Lewin M, Duclos-Vallee JC. Do the epidemiology, physiological mechanisms and characteristics of hepatocellular carcinoma in HIVinfected patients justify specific screening policies? AIDS. 2014; 28:1379-1391.

6. van Rensburg HC. South Africa's protracted struggle for equal distribution and equitable access - still not there. Hum Resour Health. 2014; 12:26.

7. National Institutes of Health. Guidelines for the Use of Antiretroviral Agents in HIV-1-Infected Adults and Adolescents. https://aidsinfo.nih.gov/guidelines (accessed May 5, 2015).

8. Batista G, Buve A, Ngom Gueye NF, Manga NM, Diop MN, Ndiaye K, Thiam A, Ly F, Diallo A, Ndour CT, Seydi M. Initial suboptimal CD4 reconstitution with antiretroviral therapy despite full viral suppression in a cohort of HIV-infected patients in Senegal. Med Mal Infect. 2015; 45:199-206.

9. Samuel R, Bettiker R, Suh B. Antiretroviral therapy 2006: Pharmacology, applications, and special situations. Arch Pharm Res. 2006; 29:431-458.

10. Manfredi R, Sabbatani S. A novel antiretroviral class (fusion inhibitors) in the management of HIV infection. Present features and future perspectives of enfuvirtide (T-20). Curr Med Chem. 2006; 13:2369-2384.

11. Calza L, Manfredi R, Pocaterra D, Chiodo F. Risk of premature atherosclerosis and ischemic heart disease associated with HIV infection and antiretroviral therapy. J Infect. 2008; 57:16-32.

12. Hester EK. HIV medications: An update and review of metabolic complications. Nutr Clin Pract. 2012; 27:5164.

13. Hazuda DJ. HIV integrase as a target for antiretroviral therapy. Curr Opin HIV AIDS. 2012; 7:383-389.

14. Davies MA, Ford N, Rabie H, Fatti G, Stinson K, Giddy J, Tanser F, Technau KG, Sawry S, Eley B, Wood R, Mofenson LM, Keiser O,Boulle A. Reducing CD4 monitoring in children on antiretroviral therapy with virologic suppression. Pediatr Infect Dis J. 2015; 12:250255.

15. Kasahara TM, Hygino J, Andrade RM, Monteiro C, Sacramento PM, Andrade AF, Bento CA. Poor functional immune recovery in aged HIV-1-infected patients following successfully treatment with antiretroviral therapy. Hum Immunol. 2015; 15:299-303.

16. Kolber MA, Saenz MO, Tanner TJ, Arheart KL, Pahwa $\mathrm{S}$, Liu H. Intensification of a suppressive HAART regimen increases CD4 counts and decreases CD8+ T-cell activation. Clin Immunol. 2008; 126:315-321. 
17. Pinzone MR, Di Rosa M, Cacopardo B, Nunnari G. HIV RNA suppression and immune restoration: Can we do better? Clin Dev Immunol. 2012; 2012:515962.

18. Nakanjako D, Ssinabulya I, Nabatanzi R, Bayigga L, Kiragga A, Joloba M, Kaleebu P, Kambugu AD, Kamya MR, Sekaly R, Elliott A, Mayanja-Kizza H. Atorvastatin reduces T-cell activation and exhaustion among HIVinfected cART-treated suboptimal immune responders in Uganda: A randomised crossover placebo-controlled trial. Trop Med Int Health. 2015; 20:380-390.

19. Gallant JE, DeJesus E, Arribas JR, Pozniak AL, Gazzard B, Campo RE, Lu B, McColl D, Chuck S, Enejosa J, Toole JJ, Cheng AK, Study G. Tenofovir DF, emtricitabine, and efavirenz vs. zidovudine, lamivudine, and efavirenz for HIV. N Engl J Med. 2006; 354:251260.

20. DeJesus E, Herrera G, Teofilo E, Gerstoft J, Buendia $\mathrm{CB}$, Brand JD, Brothers CH, Hernandez J, Castillo SA, Bonny T, Lanier ER, Scott TR, Team CNAS. Abacavir versus zidovudine combined with lamivudine and efavirenz, for the treatment of antiretroviral-naive HIVinfected adults. Clin Infect Dis. 2004; 39:1038-1046.

21. Huttner AC, Kaufmann GR, Battegay M, Weber R, Opravil M. Treatment initiation with zidovudinecontaining potent antiretroviral therapy impairs CD4 cell count recovery but not clinical efficacy. AIDS. 2007;
21:939-946.

22. Domingo P, Cabeza Mdel C, Torres F, Salazar J, Gutierrez Mdel M, Mateo MG, Martinez E, Domingo JC, Fernandez I, Villarroya F, Ribera E, Vidal F,Baiget M. Association of thymidylate synthase polymorphisms with acute pancreatitis and/or peripheral neuropathy in HIV-infected patients on stavudine-based therapy. PLoS One. 2013; 8:e57347.

23. Li T, Wu N, Dai Y, Qiu Z, Han Y, Xie J, Zhu T, Li Y. Reduced thymic output is a major mechanism of immune reconstitution failure in HIV-infected patients after long-term antiretroviral therapy. Clin Infect Dis. 2011; 53:944-951.

24. Le T, Wright EJ, Smith DM, He W, Catano G, Okulicz JF, Young JA, Clark RA, Richman DD, Little SJ, Ahuja SK. Enhanced CD4+ T-cell recovery with earlier HIV-1 antiretroviral therapy. N Engl J Med. 2013; 368:218-230.

25. Nakanjako D, Kiragga A, Ibrahim F, Castelnuovo B, Kamya MR, Easterbrook PJ. Sub-optimal CD4 reconstitution despite viral suppression in an urban cohort on antiretroviral therapy (ART) in sub-Saharan Africa: Frequency and clinical significance. AIDS Res Ther. 2008; 5:23.

(Received August 11, 2015; Revised October 13; 2015; Accepted October 14, 2015) 